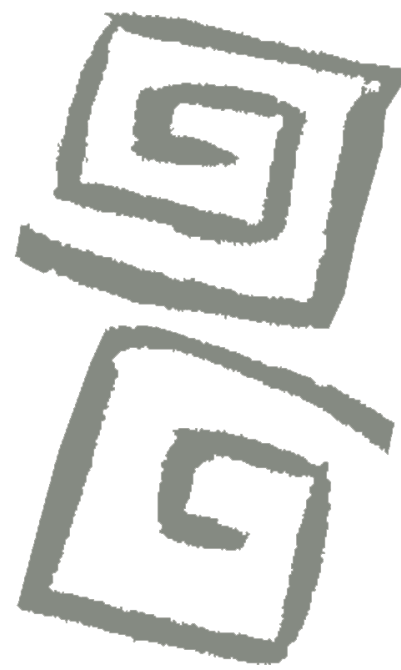

\title{
Haciendo, planeando, "planhaciendo": una experiencia de pragmatismo municipal en la pandemia del Covid-19 en cooperación con la universidad
}

\author{
Doing, planning, "planning-doing:" a case of municipal \\ pragmatism in collaboration with a university during the \\ COVID-19 pandemic
}

Helvo Slomp Junior ${ }^{1}$, Karla Santa Cruz Coelho², Delba Machado Barros ${ }^{3}$, Tulio Batista Franco ${ }^{4}$, Kathleen Tereza da Cruz ${ }^{5}$

${ }^{1}$ Autor de correspondencia. Doctor en Medicina. Profesor adjunto, Universidade Federal do Río de Janeiro, Macaé, Rio de Janeiro, Brasil. $\triangle$ id

${ }^{2}$ Doctora en Salud Colectiva. Profesora asociada, Universidade do Rio de Janeiro, Rio de Janeiro, Brasil. $\triangle$ iD

${ }^{3}$ Doctora en Salud Pública. Coordinadora Planejamento e Gestão em Saúde, Secretaria Municipal de Saúde Prefeitura de Quissamã, Rio de Janeiro, Brasil. $\triangle$ iD

${ }^{4}$ Doctor en Salud Colectiva Profesor asociado, Universidade Federal Fluminense, Rio de Janeiro, Brasil. $\triangle$ i iD

${ }^{5}$ Doctora en Medicina. Profesora adjunta, Universidade Federal de Río de Janeiro, Rio de Janeiro, Brasil. $\triangle$ iD
RESUMEN Este artículo problematiza las posibilidades municipales de hacer frente a la pandemia de COVID-19, a partir de la cooperación técnico-científica entre un municipio y una universidad del norte del estado de Rio de Janeiro, a partir de abril de 2020, que involucró la implementación de una sala de situación, procesamiento y análisis de datos para la toma de decisiones y de información para la población, centro de televigilancia, educación permanente con equipos territoriales de atención y estudio epidemiológico de COVID-19 en el municipio, entre otras acciones. En este análisis se utilizó como soporte conceptual una visión micropolítica de los conceptos de experiencia, pragmatismo, trabajo vivo en acto y deseo. La noción de "planhaciendo" se retomó como una planificación inventiva que solo puede ser narrada a posteriori, un acto imperativo, un gobierno vivo en acto que depende de un movimiento anhelante orientado por la vida, y que solo se da en espacios colectivos de prácticas de gestión y de atención a la salud.

PALABRAS CLAVES Planificación en Salud; COVID-19; Gobierno Municipal; Práctica Integral de Atención; Investigación Participativa Basada en la Comunidad; Brasil.

ABSTRACT This article critically analyzes local governments' abilities to face the COVID-19 pandemic by examining an instance of technical-scientific cooperation between a municipality and a university located in the northern Rio de Janeiro (state) beginning in April 2020. This collaboration included: the implementation of a situation room, data processing and analysis for decision making and for public communication, a telemonitoring center, ongoing training with territorial healthcare teams, and an epidemiological study of COVID-19 in the municipality, among other actions. We situate our analysis within a conceptual framework that adopts a micropolitical view of concepts such as experience, pragmatism, "live work in action," and desire. The notion of "planningdoing" is deployed as an inventive form of planning that is only narrated a posteriori, as an imperative act, a live government in action that depends on the movement of desire oriented by life, and that only takes place in collective spaces of management practices and health care.

KEY WORDS Health Planning; COVID-19; City Government; Integral Healthcare Practice; Community-Based Participatory Research; Brazil. 


\section{INTRODUCCIÓN}

A partir de los primeros meses de 2020, la pandemia de COVID-19 impuso una realidad aún más crítica al Sistema Único de Salud (SUS) en Brasil, que ya sufría los efectos de un progresivo desfinanciamiento desde la aprobación de un techo de gastos para salud y educación, en diciembre de 2016, que entró en vigor a partir de $2018^{(1,2)}$. En paralelo, las universidades públicas, aunque también fueron blanco del mismo proceso, inmediatamente se sumaron a colaborar con el SUS y la sociedad brasileña para enfrentar la pandemia, y se multiplicaron las experiencias de cooperación entre estas instituciones y diversos municipios brasileños ${ }^{(3,4)}$.

Aunque en su alcance legal e institucional la gestión del SUS sea tripartita y las instancias federal, estadual y municipal sean corresponsables en cada territorio, durante la pandemia, el papel de los municipios y de sus entidades representativas parece haber cobrado un protagonismo aún más evidente. Cabe a esta instancia de gestión conducir los servicios de salud $-y$ de todos los otros sectores de la acción pública- que deben dar cuenta tanto de las demandas previas a la pandemia -como, por ejemplo, de las enfermedades crónicas no transmisibles- como de la atención a casos sintomáticos respiratorios y sospechosos de COVID-19, además de acciones de vigilancia en salud para contener la propagación de la enfermedad, entre otras. Tal conjunto de demandas exige decisiones secuenciales de acuerdo a los recursos disponibles y a la lectura posible de la realidad que se presenta ante el avance de la pandemia ${ }^{(5)}$.

Sin embargo, para que cada gestión municipal pueda enfrentar cotidianamente un escenario agravado por la pandemia, se sabe que es indispensable, entre otras cosas, el apoyo articulado y coordinado de forma interfederativa -atribución de las esferas estadual y federal del SUS- que posibilita tanto una comprensión mutua en la lectura de los problemas que se plantean, como una acción conjunta. Desde nuestra perspectiva, los municipios contaron con un conjunto de normas y de información, a veces contradictorias y frágiles, y cuando hubo incentivos financieros, indujeron a prácticas no consensuadas como, por ejemplo, centralizar las acciones en instancias como la hospitalaria y los centros de admisión/testeo en los primeros meses de la pandemia. Al inducir la centralización de las acciones en esas áreas especializadas -también necesarias para garantizar la integralidad del cuidado en la pandemia- se restringió el incentivo estratégico a las acciones territoriales, lo que llevó a desestimar la significativa capacidad instalada de la red del primer nivel de atención en Brasil, además de exponer municipios con menor estructura hospitalaria a un menú reducido de respuestas ${ }^{(6)}$.

Uno de los fenómenos que acompañó el enfrentamiento brasileño de la pandemia es el negacionismo presente en sectores del gobierno federal, usado como estrategias políticas para priorizar las actividades económicas en detrimento de estrategias de protección como el distanciamiento social ${ }^{(7)}$. Todo este escenario impuso a los municipios (y a los estados) una perspectiva a veces solitaria de intervención, que los llevó a tomar actitudes muchas veces aisladas. En este artículo se abordan aspectos de una de esas experiencias municipales que se están llevando a cabo.

Al norte del estado de Rio de Janeiro, un grupo de profesores vinculados al campus Macaé de la Universidad Federal del Rio de Janeiro (UFRJ/Macaé), en abril de 2020 -en los inicios de la pandemia en Brasil- organizó una iniciativa para apoyar las redes de atención de la salud, para lo cual creó un grupo de trabajo multidisciplinario para enfrentar el COVID-19 (GT COVID-19 UFRJ/ Macaé $)^{(8,9,10,11)}$. Esta cooperación interinstitucional se encuentra consolidada, en especial, junto a algunos municipios como, por ejemplo, el municipio de Quissamã, con la finalidad de fortalecer las inevitables e intempestivas respuestas que exige la pandemia, no siempre acordes a la capacidad fiscal disponible al momento de su eclosión ${ }^{(12,13)}$. En el caso del municipio de Quissamã, aunque varios años antes de la pandemia ya era un 
escenario histórico de colaboración con la UFRJ/Macaé en varias actividades, en aquel momento enfrentaba solo las primeras semanas pandémicas con acciones que describimos en el siguiente apartado, haciendo hincapié en aquellas que fueron fruto de nuevas colaboraciones.

La colaboración entre universidades y municipios existe hace mucho tiempo en Brasil. Por ejemplo, en la década de 1990, un grupo de profesores organizó el Laboratorio de Planificación y Administración de Sistemas de Salud (LAPA) en el Departamento de Medicina Preventiva y Social de la Universidad Estadual de Campinas (UNICAMP), por medio del cual desarrolló proyectos junto a diversas redes municipales de salud ${ }^{(14,15)}$. En ese ciclo de experiencias se formularon muchos de los acuerdos tecnológicos para la gestión y el cuidado en salud que fueron estratégicos en la configuración del SUS, que significaron una innovación en este tipo de relación interinstitucional. Se trata de un esfuerzo por quebrar la polaridad entre "quien produce el conocimiento (universidad)" y "quien lo pone en práctica (municipio)", para pasar a una relación de "hacer en conjunto", simétrica. Así como en la tradición de la producción del LAPA, en la experiencia aquí problematizada se buscó pensar y experimentar las posibilidades en conjunto, unos enseñando a otros, unos aprendiendo junto a otros.

El trabajo conjunto entre un grupo de profesores y estudiantes de la universidad, y trabajadores de una red de servicios de salud, observa algunos presupuestos importantes. Primero, que los diferentes grupos traen consigo diferentes "cajas de herramientas" para lidiar con situaciones complejas como la pandemia, produciendo un encuentro entre saberes acumulados a lo largo de muchos años de experiencia junto a los servicios de salud, y estudios relacionados al mundo del trabajo y cuidado en salud.

La noción de caja de herramientas, propuesta por los filósofos Michel Foucault y GiIles Deleuze, implica que conceptos y teorías solo tienen sentido si son relevantes frente a problemas concretos y singulares, que pueden incluso demandar nuevos conceptos y teorías. Tal proposición fue incorporada en la salud colectiva brasileña a fines de la década de 1990, por Emerson Merhy ${ }^{(16,17,18)}$.

Reconocer que la "ciencia" también tiene como componente el saber de la experiencia remite al segundo presupuesto: que esta relación debe ser simétrica, con espacios de diálogo y escucha entre los grupos que se proponen cooperar. Por último, el producto final será siempre el resultado de los espacios intercesores que se producen en el encuentro $^{(19)}$ entre los diversos actores de la escena del cuidado para enfrentar el COVID-19. Estos presupuestos demandan ciertas tecnologías de gestión del cuidado, que han sido requeridas en la cooperación universidadmunicipio como un modo de actuar en y con las redes de servicios de salud.

El presente artículo es una problematización en torno a las posibilidades municipales frente a la pandemia por el COVID-19 en Quissamã (Río de Janeiro), en el marco de la cooperación técnico-científica con la UFRJ/ Macaé. El objetivo es reflexionar, a partir de la experiencia de esta cooperación, y hasta el momento en que finalizamos este artículo, de qué modo las prácticas sanitarias y de gobierno, en el ámbito municipal, se constituyen en una experiencia pragmática de lo posible.

\section{Breve descripción de las acciones municipales durante la pandemia}

El municipio de Quissamã tiene 24.700 habitantes y está localizado en la región norte fluminense $\mathrm{e}^{(20)}$. Al iniciar las acciones para enfrentar la pandemia de COVID-19, en abril de 2020, se crearon varios dispositivos, entre ellos: incremento de los programas sociales para familias que se encuentran en riesgo social (ancianos, personas con discapacidades y de bajos ingresos); aporte nutricional para escolares de la red municipal con ayuda económica y entrega de canastas de la agricultura familiar; higienización sanitaria urbana; barreras sanitarias en los límites territoriales municipales; implementación de hospitales de campaña; telemonitoreo de los 
sintomáticos respiratorios, y casos confirmados con tratamiento domiciliario; integración entre servicios del sector salud, con especial atención a usuarios con condiciones crónicas y sufrimiento mental; redefinición de camas clínicas y unidades de terapia intensiva para atender los casos de COVID-19 en el hospital municipal; teleatención psicológica para la población y las y los trabajadores; atención telefónica Disque-Salud y, de ser necesario, atención domiciliaria de los casos sospechosos; plan de información, comunicación y educación en salud para enfrentar el COVID-19 con vehículos con altavoces en el territorio, divulgación de medidas de protección en las redes sociales y acciones de educación permanente y continua en salud junto a los servicios de salud ${ }^{(12,21,22)}$.

Los aportes financieros del gobierno federal y estadual se destinaron a la adquisición de equipos de protección individual e insumos relacionados con la asistencia a usuarios con COVID-19; a la implementación y funcionamiento del Centro de Triage Respiratorio (CTR) y al pago de personal ${ }^{(23,24,25,26,27,28)}$. Además de atender a la población propia, el municipio actuó de forma integrada a la región, manteniendo el acceso a usuarios de municipios vecinos al CTR, a las camas clínicas y de terapia intensiva COVID-19 del hospital municipal (habilitadas por el Ministerio de Salud), y a la maternidad municipal, dado que el municipio ya era referencia regional para el parto de bajo riesgo ${ }^{(12,21,22,29)}$, a lo que se sumó el legado del SUS, que hizo la diferencia durante la pandemia.

En Quissamã ya se estaban organizando varios frentes de trabajo cuando, en el mes de marzo de 2020, algunas de las primeras ofertas de trabajo del GT COVID-19 UFRJ/ Macaé comenzaron a divulgarse por los municipios de las regiones Norte Fluminense y Baixada Litorânea del estado del Rio de Janeiro, y otros actores regionales adhirieron a la propuesta.

En el comienzo de la pandemia, las fragilidades en la articulación interfederativa no permitieron el debate sobre estas cuestiones por la velocidad de respuesta que exigía la realidad. Así, se propuso un foro de discusión plural, no institucional, creado y coordinado por el GT COVID-19 UFRJ/Macaé en encuentros online con gestores, trabajadores, comunidad universitaria, movimientos sociales y otros, espacio que se denominó Fórum dos Territorios ${ }^{(10,11)}$. Las cuestiones planteadas se agruparon en cuatro grandes temas: gestión y redes de atención en salud, proceso de trabajo, bioseguridad, educación en salud /educación permanente en salud.

Con la firma del acuerdo de cooperación entre la intendencia de Quissamã y la UFRJ/ Macaé, surgieron otras acciones en cooperación, como implementar el Panel COVID-19 (ver más adelante), procesar y analizar los datos municipales a partir de las mejores fuentes disponibles para producir información y evidencias necesarias para la toma de decisiones, divulgar la situación del COVID-19 y de otro tipo de información para la población. Además, se comenzaron a realizar entrevistas en vivo en plataformas de Internet, acciones de educación permanente en salud junto a las equipos territoriales de cuidado en salud y estudios de base poblacional para el cálculo de la prevalencia de la enfermedad en el municipio, entre otras nuevas acciones ${ }^{(10,13)}$.

\section{Breve descripción de la iniciativa universitaria que se sumó al movimiento municipal}

Como se describió en la introducción de este artículo, ante la magnitud y complejidad del problema sanitario causado por la pandemia de COVID-19, la universidad se movilizó para contribuir con las redes de servicios locales-regionales de salud e intersectoriales. Inicialmente una iniciativa de docentes de la institución, se ramificó en una red multidisciplinar e interinstitucional al establecer colaboraciones internas y externas a la institución, enfocadas en la región, como por ejemplo la colaboración con el Campus de Rio das Ostras de la Universidad Federal Fluminense ${ }^{(10,11)}$. Allí se produce el encuentro de dos mundos (universidad y servicios), los cuales, a partir de sus diferentes perspectivas, comienzan a trabajar juntos frente al desafío 
de proteger y cuidar a la población de la amenaza del nuevo coronavirus.

El GT COVID-19 UFRJ/Macaé se organiza en subgrupos de trabajo a partir de cinco ejes $^{(10)}$ :

1) Monitorear la pandemia y la gestión en salud (información epidemiológica, tecnologías para implementar salas de situación con datos en tiempo real); central de telemonitoreo para usuarios que requieran aislamiento domiciliario; foro territorial en las regiones Norte Fluminense y Baixada Litorânea del estado de Rio de Janeiro, para enfrentar la pandemia de COVID-19; soporte de tecnología de información.

2) Apoyar a los equipos asistenciales en el territorio de cuidado (desarrollar acciones de educación permanente en salud vinculadas al cuidado en salud, protocolos clínicos y procedimientos operacionales estandarizados para la asistencia hospitalaria; identificar factores de riesgo nutricionales y otros asociados al COVID-19 e incentivar la práctica de actividades físicas durante la cuarentena; realizar acciones de información en seguridad biológica, laboratorio para la realización de exámenes de proteína C-reactiva/PCR para COVID-19 y acciones en salud mental).

3) Divulgar las actividades del GT COVID-19 UFRJ/Macaé y la información científica, elaborar material educativo e informativo para combatir las fake news, crear teleatención para la población del municipio de Macaé).

4) Realizar acciones de protección social (seguridad alimentaria y nutricional, ayuda humanitaria para grupos de personas y poblaciones en situación de emergencia en cuanto a la vulnerabilidad socioeconómica o alto riesgo de contagio).

5) Desarrollar investigación básica relacionada con el COVID-19 (investigaciones de nuevos medicamentos contra SARS-Cov-2 a través de bioinformática, herramientas moleculares in vitro e in silico para estudios epidemiológicos, tratamiento y diagnóstico laboratorial de COVID-19).
Estos frentes de trabajo actuaron con autonomía, pero de modo articulado entre sí ${ }^{\prime(10)}$. La gama de acciones puestas a disposición por la universidad, que enumeraremos más adelante, y la combinación entre ellas, dependió y depende de las necesidades de cada municipio y de la disponibilidad de la carga horaria docente. El trabajo dio como resultado diversos productos, que incluyeron un seguimiento interinstitucional semanal de la pandemia en la región, con especial énfasis en los municipios que establecieron colaboración técnico-científica con la universidad.

\section{Elección del material empírico y del ángulo analítico}

Está demás decir que hay una gran amplitud de presentaciones posibles a partir de las múltiples actividades relacionadas con este trabajo, cada uno apunta a diferentes áreas del conocimiento y con metodologías propias de esta propuesta que representó y aún representa una experiencia importante para todas las personas involucradas, de la que se podrán obtener otros análisis pero, sobre todo, más resultados concretos para la población, que busca el cuidado en salud.

Para este artículo, nos esforzamos en observar la experiencia de este municipio a partir de la gestión de sus redes de salud para enfrentar la pandemia y en cooperación con la universidad. No pretendemos aquí evaluar sobre la base de modelos preestablecidos y considerados como un estándar, sino abrir debates entre estas y otras experiencias y reflexiones.

En los debates que tuvieron lugar en el marco de la colaboración emergieron una serie de preguntas que dispararon la problematización y la construcción de este artículo: “¿cuál es el papel de la atención primaria en tiempos de pandemia?"; "¿cómo garantizar la continuidad del cuidado a otras condiciones no-COVID?"; "¿Cómo podrían las evidencias científicas cualificar la atención de usuarios con COVID-19 confirmado o no?"; "¿de qué modo se puede mejorar la organización de los flujos asistenciales durante la pandemia?". 
Estas preguntas se ramifican en otras, de orden epistemológico: las personas involucradas (docentes/estudiantes/investigadores de la universidad, trabajadores de la gestión y de los servicios de asistencia de las redes municipales de salud y usuarios) ¿a qué planos de la experiencia consiguen acceder, en situaciones así?"; "¿de qué modo podemos problematizar tales experiencias al punto de requerir conceptos, para lograr una construcción teórica que, producto de la experiencia, pueda ayudar a comprenderlas?".

En este artículo intentamos evitar tomar la experiencia como una realidad objetiva que deberíamos, ante todo, registrar objetivamente y con fidelidad, para después interpretar. Otros estudios, nuestros y de colegas, ya tomaron y toman para sí esta tarea. Además, varios trabajos han estudiado y publicado datos sobre la pandemia de la región de Macaé. Por ejemplo, Guimarães et al. delinearon un panorama de escenarios epidemiológicos posibles para el municipio, con estimaciones de infectados, hospitalizados, casos graves y defunciones ${ }^{(30)}$; Sperandio et al. registraron el escenario municipal al inicio de la pandemia $^{(11)}$, además de notas técnicas sobre simulaciones epidemiológicas ${ }^{(31)}$; Branco et al. ${ }^{(31,32)}$ documentaron el desarrollo de una solución tecnológica para visualizar en tiempo real, vía Internet, la evolución de los dados epidemiológicos sobre COVID-19 de los municipios del Norte Fluminense; Guimarães et al. analizaron la mortalidad por COVID-19 en las diez semanas iniciales de la pandemia en Macaé, y la mortalidad comparada en los cuatro mayores municipios del Norte Fluminense y de la Baixada Litorânea ${ }^{(30,31)}$; Chaves et al. propusieron un sistema de organización de telemonitoreo de personas en aislamiento domiciliario indicado por la Vigilancia en Salud del municipio, oriundas de la red pública y/o privada ${ }^{(33)}$; entre otros productos del GT COVID-19 UFRJ/Macaé.

Así, al mismo tiempo que los estudios mencionados estaban en curso, recuperamos la experiencia de la cooperación universidadmunicipio desde su condición inmanente, nos esforzamos por experimentar la experiencia, y es lo que pretendemos relatar en este texto. Recuperamos retrospectivamente detalles que dejan entrever esos otros análisis ya citados que hemos hecho sobre la misma experiencia, conscientes de que será un ejercicio parcial y limitado. A continuación, explicitaremos esa perspectiva conceptual.

\section{Herramientas metodológicas y maquinaria conceptual}

La estrategia de entrada al campo y la recolección de datos, con relación al recorte delineado en la sección anterior, fue rememorar la experiencia de algunos de nosotros ante la experiencia del otro, mediante reuniones frecuentes y grupos de trabajo a distancia, por Internet. Siguiendo a Jorge Larrosa Bondía, entendemos experiencia como lo que nos pasa, nos sucede y/o nos toca (y no solo lo que pasa, sucede o toca), la tensión entre placer, pasión y libertad, dolor y miedo ${ }^{(34)}$.

Se pretendió dar visibilidad a ciertos problemas que atraviesan los estudios e intervenciones, para proponer nuevas preguntas, habilitar una perspectiva de análisis diferente sobre el fenómeno pandémico como un todo para los municipios, y pensar otras posibilidades para enfrentarlos. En esta reflexión retomamos la misma experiencia, pero desde otra mirada, que solo es posible con lentitud, delicadeza y paciencia ${ }^{(34)}$, logrando nuevas aproximaciones que en otros momentos de las acciones y de la investigación no tenían sentido.

Tal análisis pide un tratamiento en el mismo sentido, y para este trabajo optamos por conceptos-herramienta que encontramos en el pragmatismo de William James, retomado por David Lapoujade ${ }^{(35)}$ : nuestro énfasis estará en la práctica, en las acciones, en las relaciones. Evitamos la construcción de verdades a partir de modelos preexistentes porque nos interesa percibir dónde y cuándo se desarrollaban ideas nuevas en esta experiencia, ideas que en esta perspectiva teórica equivalen a acciones, creaciones y cambios, realidades que hablan por sí y con las cuales intentamos conversar. 
Cuando interpretamos, intentamos evitar la pura representación cognitiva, para recuperar los esfuerzos emprendidos, las emociones y sentimientos (como el de pertenencia o de miedo, por ejemplo), pero también los modos de actuar instituidos por los hábitos que se entretejen en nuestros cotidianos. Tentamos aquí una epistemología a partir de la experimentación, un método retrospectivo que parte de la acción y de la invención para desde allí buscar las ideas que, sin mostrarse, podrían estar presentes desde el inicio, acompañando todo lo que se hizo hasta ahora.

"La idea verdadera no es solo aquello en lo que creemos, que hacemos o pensamos; es aquello que hace creer, que hace actuar o que hace pensar" ${ }^{\prime \prime 35}$. Lapoujade toma de James la idea de conocimiento deambulante para referirse a ese modo nómade de transitar relaciones y acontecimientos para construir puentes, relaciones tejidas sobre relaciones, que para el autor es un ejercicio de conocimiento constructivista: "los conceptos son cortocircuitos, abreviaturas, [...] son pedazos de experiencia condensada"(35).

Nos ocupamos de los conceptos después de definir el recorte de la experiencia, que sería nuestro material empírico, y la noción de pragmatismo, en el sentido dado por Lapoujade, como herramienta para este constructivismo. Tomamos pragmatismo aquí como evaluación práctica de la acción planteada como problema y riesgo, reflexionando sobre cuáles son las funciones de los conceptos y de la propia verdad, y como se conforman las ideas y lo que hacemos con ellas ${ }^{(35)}$. En el caso de esta reflexión, bajo la óptica de este pragmatismo puede pensarse tanto la acción municipal sobre la pandemia, como las propias formas de pensar esa acción municipal.

Es en ese sentido que retomamos el pragmatismo propuesto por el LAPA/UNICAMP de la década de 1990 en Brasil, que citamos en la introducción ${ }^{(14,15)}$ : el recién nacido SUS, replicado a una estructura federativa como la brasileña, requería de una gestión diferente, y la planificación -especialmente en las experiencias de cooperación de la universidad con municipios- se destacó como andamiaje tecnológico importante para muchos autores.
Sin embargo, había una tensión entre la planificación Ilamada normativa o prescriptiva, que impone verticalmente sus métodos, aunque imbuida de una acción reformadora, y el pensamiento estratégico que busca la producción de sujetos reformadores ${ }^{(36)}$ :

Por un lado, están los que abogan una prevalencia del método de la planificación sobre los sujetos concretos y, por otro, los que abogan lo inverso, al punto de subordinar la discusión del método a una comprensión más fundamental de los sujetos en escena y sus proyectos, hasta el límite de un planhaciendo y no de una planificación. ${ }^{(36)}$

El neologismo en portugués "fazejamento" -combinación de "fazer" (hacer) y "planejamento" (planificación), que Emerson Elias Merhy atribuye al sanitarista David Capistrano da Costa Filho, que en aquella década formaba parte de la gestión municipal de salud de Santos, municipio del estado de San Pablo- lo retomamos aquí como herramienta para problematizar las decisiones de un equipo municipal de gestión en salud sobre la pandemia. Desde nuestra perspectiva, el "planhaciendo" se refiere justamente a la acción micropolítica en la gestión y al cuidado en salud cuando la planificación a priori no es posible y/o necesita ser construida en acto. En ese sentido, la apuesta principal es el encuentro como dispositivo para producir una potencia que impulsa las acciones de cuidado. En nuestro caso, nos referimos a la acción conjunta y compartida entre profesores y estudiantes con trabajadores y usuarios de una red de atención de la salud.

La micropolítica del trabajo y del cuidado en salud nos aportó herramientas de análisis para esta experiencia, en especial el concepto de trabajo vivo en acto y de deseo. Este último lo usamos siguiendo a Gilles Deleuze y Félix Guattari, tal como es retomado por Franco y Merhy ${ }^{(37)}$ : el deseo no como falta que exige satisfacción, sino como producción permanente en la vida, energía propulsora que pone a los colectivos en acción, proceso deseante que atraviesa las diversas 
subjetivaciones en juego. De estos autores recuperamos también la noción estratégica de que el trabajo en salud -tanto en las actividades de gestión como de atención de la salud, incluso las acciones cuidadoras- solo acontece en acto y, por lo tanto, es vivo, dado que se concretiza en el instante mismo en que se está realizando, por lo que siempre es potencialmente creador ${ }^{(19,37)}$. El trabajo vivo en acto será retomado aquí sin hacer distinción entre las instancias de gestión y de atención de la salud, pero con especial atención en la primera.

\section{Aspectos éticos}

Al mismo tiempo en que el GT COVID-19 UFRJ/Macaé lleva a cabo sus acciones, coordina también el proyecto de investigación "Enfrentamento do COVID-19 na região norte fluminense e baixada litorânea: ações, perspectivas e impactos", aprobado por el Comité de Ética en Investigación bajo el número CAAE 32186520.7.0000.5699, cuyo campo de investigación es el propio trabajo del GT COVID-19 UFRJ/Macaé y que, si bien no adiciona nuevas actividades a los ejes de intervención del GT, se trata de una estrategia que aporta una dimensión analítica a esas actividades, que posibilitan también su registro científico. El análisis constante en este artículo integra los resultados parciales de esta investigación.

\section{Haciendo, planificando, planhaciendo salud}

El COVID-19 exigió respuestas rápidas y diversas de la gestión municipal de Quissamã para reducir el contagio, evitar el sufrimiento y la muerte, tanto de la población como de los profesionales de la salud, teniendo en vista la alta transmisibilidad y letalidad ampliamente documentadas. Los gestores de la salud se encontraron bajo la égida del miedo de exponerse y de exponer a todos a la contaminación, en un contexto en el que aún se conocía poco sobre la enfermedad y su tratamiento, y escaseaban los insumos para testeo, los equipos de protección individual y otros. El escenario exigía una mirada global de las experiencias exitosas en el mundo para la actuación local, es decir, en el territorio donde se produce vida y cuidado en salud. Era necesario acceder a las mejores evidencias científicas para enfrentar el COVID-19, esa fue la principal motivación del municipio al buscar la colaboración de la UFRJ/Macaé.

En Quissamã, se adoptaron acciones integradas entre los sectores de salud, asistencia social, educación, servicios públicos, comunicación y desarrollo económico, para sumar esfuerzos en el sentido de reducir la propagación del coronavirus y proteger la vida. En el área de salud, se ampliaron cuidados hospitalarios, atención psicosocial y vigilancia epidemiológica. Al inicio de la pandemia, como en muchos municipios vecinos, se suspendieron las acciones territoriales de salud familiar y vigilancia ambiental, como también se cerró la mayor parte de los servicios ambulatorios especializados, a fin mitigar el riesgo de transmisión de la enfermedad, basándose también en la capacidad municipal de aquel momento, y garantizar con rapidez los insumos necesarios para dar cuenta de las primeras recomendaciones de la Organización Mundial de Salud sobre COVID-19(38).

Atender la urgencia de la pandemia de COVID-19 y, al mismo tiempo, continuar con el cuidado de la población que necesitaba atención para sus problemas cotidianos de salud, era una tarea difícil de realizar. Exigió una mayor integración de la red de servicios y organización de las estructuras de gestión de los servicios. Para dar cuenta de esta situación, una parte del equipo de gestión se volcó a la asistencia hospitalaria y al triage respiratorio, otra buscó garantizar el cuidado en salud para otras demandas espontáneas, dando continuidad a las acciones propias de la atención básica frente al distanciamiento social. Integrar esos frentes no fue una misión imposible, dado que el diálogo y la integración entre servicios ya formaba parte del cotidiano del equipo, a través de dispositivos previamente instituidos. Sin embargo, la implementación de nuevas acciones requirió de nuevos acuerdos con la red 
municipal de salud ${ }^{(39)}$. En ese momento, en especial, la planificación fue una herramienta aglutinante entre los frentes de salud, organizando acciones compartidas entre el consejo municipal de salud, la universidad y la secretaría estadual de salud ${ }^{(39)}$, que obtuvo el apoyo también de la intendenta, que logró el compromiso de los diversos sectores y actores, configurándose el pragmatismo municipal en acto.

Un hacer de estas características tensiona cualquier planificación previa y exige incluso otra planificación, simultánea a la acción, o una planificación que solo puede ser escrita a posteriori de las acciones. La planificación se resignifica, pues se va esbozando a veces para dar respuestas de gobierno que no pueden esperar porque necesitan proteger la vida en medio de un océano de incertezas. Un actuar imperativo, un "gobierno vivo en acto", parafraseando el trabajo vivo en acto ${ }^{(19,37)}$, que no prescinde de la planificación sino que la invierte. Es necesario hacer ahora, por lo tanto, la invención y la experimentación se tornan inevitables. A esto denominamos "planhaciendo".

En la experiencia analizada, el "planhaciendo" fue productivo, y fue cobrando más y más potencia en la producción de vida. El municipio estaba solo al inicio de la pandemia y luego comenzó a caminar junto a la universidad. Si bien mantuvo sus experimentaciones, la colaboración agregó otras miradas y nuevas acciones, por ejemplo, un abordaje del conjunto desarticulado de información y directrices disponibles. Así, el análisis de los datos se colocó en el centro de las decisiones para enfrentar la pandemia, a fin de evitar ciertas distorsiones políticas de los hachos científicos que es un fenómeno actual, sin precedentes en Brasil, si se consideran sus dimensiones ${ }^{(7)}$. Se conformaron nuevas lecturas de la realidad municipal y regional y de los propios acuerdos gubernamentales en las primeras horas de la pandemia, que ya exigían nuevos análisis. Ya se contaba con el principal: un movimiento deseante $^{(37)}$ que colocaba y coloca la vida en el centro del escenario de acciones y el hacer colectivo como la gran potencia ${ }^{(40)}$.
Pero los desafíos aún se imponían. En reuniones de educación permanente en salud con los equipos asistenciales, uno de los problemas centrales fue el miedo de los trabajadores a contagiarse, contagiar a colegas $y$ familiares, y de morir por COVID-19, un sentimiento que se manifestaba en el cotidiano, incontrolable y a veces paralizante ${ }^{(10)}$. El escenario general de la pandemia, asociado al miedo, afecta de tal forma a las personas, que produce siempre algún tipo de sufrimiento mental, y la constatación de este hecho coloca en escena otro conjunto de preocupaciones: la salud mental percibida como una crisis silenciosa dentro de la crisis sanitaria, muchas veces es un problema invisibilizado ante la urgencia de enfrentar el COVID-19, pero igualmente agresivo y dañino(40).

No cabe detallar en este artículo cómo se trabajó este problema en particular, pero ilustra, juntamente, cómo en la incertidumbre de la información que circulaba entre profesionales de salud y usuarios confluían elementos desestabilizantes del trabajo en salud, tanto de gestión o de atención. Fue necesario rever las actividades programadas para cada día y, mientras se hacía lo que se necesitaba hacer, se evaluaban las acciones en tiempo real en debates interinstitucionales en el marco de la cooperación universidadmunicipio, que ocupó parte del tiempo de las propias acciones. Hoy entendemos que allí se estaba produciendo el "planhaciendo" del gobierno y de los equipos, conformado por diferentes miradas.

La presión del escenario pandémico y la apertura a nuevos pensares/saberes/actuaciones permitió que los espacios colectivos que incluían actividades de educación permanente en salud -algunas de las cuales, en colaboración con la universidad- fueran algo más que solo dispositivos para compartir decisiones administrativas y nuevas normas, y se tornaran verdaderos espacios de formulación de gobierno. Por momentos se produjo un actuar colectivo y deseante que, sin esperar soluciones fáciles o "mágicas", fue tejiendo por todos lados una coautoría intra y entre equipos, que algunos de nosotros deno-

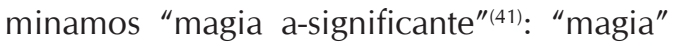


porque no es un trueque, prestidigitación, ni tampoco se origina de un Yo que irradia a los otros lo que debe ser hecho, sino una transmutación de las relaciones, cambio que emerge de un Nosotros en franca alianza por la vida; y "a-significante", porque antecede a nuestra representación cognitiva sobre esta transmutación, el pensar y el actuar acontecen al mismo tiempo.

¿Cómo esto es posible, en plena curva de desguace del SUS, sistema creado en un país condenado a un permanente "estado de sitio económico"(2)? La potencia de la esfera municipal del SUS, incluso en medio de una tempestad, no es novedad. Se manifestó antes de la creación del SUS, ya a mediados de la década de 1970, incluso antes ${ }^{(42)}$. Como uno de los principales protagonistas del movimiento de la reforma sanitaria y motor que impulsó la construcción del sistema, el movimiento denominado "municipalista" colocó al municipio como un protagonista importante en la gestión del sistema de salud, con atribuciones operativas significativas sobre el SUS, y aportó experiencias reales al diseño de buena parte de lo que se implementaría en el SUS en las décadas siguientes. Por lo tanto, no es novedad el buen "planhaciendo" experimental que se anticipó a los avances que se produjeron en la salud pública brasileña, aunque siempre haya sido saboteado por los problemas de financiamiento que acompañaron al SUS hasta los días previos al ataque de la Enmienda Constitucional (EC 95), que congeló la inversión estatal en políticas sociales y educativas, un golpe al sistema que se consideró fatal ${ }^{(1,2)}$.

Las respuestas municipales, como las que estamos discutiendo aquí, son posibles porque ya había un legado del SUS con una red municipal madura que, aunque asfixiada por un desfinanciamiento sistémico, es la mayor garantía de la población en tiempos pandémicos. Quissamã es una de las raras excepciones brasileñas, al ser un municipio que, hasta el momento en que finalizamos este artículo, contaba con el $100 \%$ de cobertura de salud familiar ${ }^{(43)}$, un patrimonio estratégico para enfrentar la pandemia; pero que también, en los primeros meses de la pandemia, replegó las acciones de atención primaria, en función de ciertas normativas e instrucciones, como ya mencionamos ${ }^{(26,28,38)}$. En los primeros encuentros entre los equipos de gestión y atención de Quissamã y el GT COVID-19 UFRJ/Macaé, hubo un pedido claro de problematizar esta situación, que fue uno de los primeros ejes de trabajo de la cooperación. El consenso que se fue formando es que el territorio municipal es el lugar por excelencia de los cuidados de proximidad( ${ }^{(6)}$, una proximidad no solo geográfica, sino también afectiva, una disposición capilarizada de cuidados sociales y en salud que aumenta la capacidad de impacto en la pandemia ${ }^{(6)}$.

Por un lado, esa proximidad multidimensional posibilitó acciones de vigilancia en el territorio, como apertura de espacios de conversación sobre temas que paradójicamente eran $y$, aunque ahora menos, aún son polémicos, en la población, como el distanciamiento social, el uso de máscaras y otras medidas preventivas; $y$, por otro lado, puso en revisión permanente los flujos locales de las redes y la integración entre diversos sectores y el área de salud, en el sentido de reforzar el pensamiento epidemiológico. Al mismo tiempo, formaron parte de las acciones las medidas de impacto social y el esfuerzo por un movimiento coordinado, incluso con eventuales diferencias en la interpretación de los acontecimientos y en las propuestas de nuevas acciones restringidas a las fronteras municipales, de hecho, no respetadas por el SARS-Cov-2. Varias de estas acciones comprometían, al mismo tiempo, al municipio y la universidad, que se encontraban varias veces por semana, en una alianza por la vida que también relacionamos al concepto de magia a-significante, ya mencionado.

Proponemos pensar el "planhaciendo pragmático" centrado en la vida como un esfuerzo por forjar acciones contracorriente, incluso con resultados improbables, para que el SUS pueda alcanzar, localmente, algunos de sus objetivos, contradiciendo proyectos de austeridad económica y financiera como la Enmienda Constitucional 95. Ese "andar juntos" entre sujetos que comparten cotidianos similares, conocimientos, necesidades, 
deseos, incertezas, miedos y otros sufrimientos, cuando la vida está en juego, ganó precedencia en esta experiencia, y nos mostró que lo posible puede ser siempre mayor de lo que parece inicialmente. Creemos que la colaboración entre universidades y municipios, con relaciones simétricas y decisiones compartidas, está entre los más potentes pragmatismos para hacer vivir a las personas y al SUS, y que emergieron mucho antes de que exista nuestro sistema. Desde nuestra perspectiva, representa claramente la apuesta por un proyecto ético-estético-político ${ }^{(41)}$ que se manifiesta en la insistencia de inventar para existir, desobedeciendo ciertas matemáticas económicas perversas que no consideran la vida de las personas o de las comunidades como un bien innegociable.

\section{CONSIDERACIONES FINALES}

En el ámbito de la comunidad académica, experimentar el enfrentamiento vivo de la pandemia junto con los equipos de gestión y atención municipales desplegó un gran aprendizaje, un desplazamiento hacia otro territorio: el lugar de la defensa de todas las vidas como imperativo ético de la producción de conocimiento; o el lugar de un hacer que depende de escuchar y cooperar, como acciones constitutivas de investigar. Como dijo un docente del GT: "los estudiantes se responsabilizaron junto a nosotros de acciones y proyectos que tienen una gran relevancia para salvar vidas, y eso ha sido un aprendizaje muy importante para todos nosotros. Aprendemos haciendo el papel social de la universidad, y estamos orgullosos de eso".

Cabe destacar, además, que el reconocimiento por parte del municipio de la potencialidad de la universidad respecto de la cooperación, produjo un gran agenciamiento en su cuerpo social, canalizando deseos de actuar frente a la pandemia con acciones concretas que se efectivizaron en ese "planhaciendo" compartido, y en varios "productos" desarrollados específicamente para atender los problemas que se presentaban en el ámbito de la acción real del municipio, multiplicando los espacios de reflexión, de producción de conocimiento, de reconocimiento mutuo y de valorización recíprocas. De esos encuentros emergieron deseos de continuar realizando colaboraciones que fortalezcan el SUS, tanto aquellas relacionadas con el registro y la problematización del "planhaciendo en acto", como con la recolección y el procesamiento a posteriori de lo ya hecho, como también aquellas que otorgan sentido a la producción de conocimientos para tomar las mejoras decisiones posibles. Por supuesto, dentro de las posibilidades institucionales, cada vez más amenazadas en la actualidad.

La capilaridad de nuestro sistema público de salud es de suma relevancia para los principios y las directrices del SUS, como universalidad, equidad, atención integral a la salud, regionalización, jerarquización y descentralización, y se torna aún más importante ante la propagación del COVID-19. El pragmatismo municipal, en colaboración con la universidad, en esa unión entre salud-educacióninvestigación-extensión, aun con la fragilidad actual del SUS, ayuda a salvar vidas y protege a las más vulnerables, aunque en Brasil se siguen contabilizando muertes que tal vez pudieron ser evitadas. Tal cooperación puede producir ciertos acuerdos tecnológicos a partir de la relación universidad-servicio que potencializan el "planhaciendo" municipal como saber de la práctica y, por lo tanto, válido, siempre que medien relaciones simétricas, tanto en la producción del conocimiento como en las intervenciones, y en los modos de gestionar la salud y producir el cuidado. 


\section{AGRADECIMIENTOS}

A las y los colegas docentes, estudiantes y trabajadores del área de la salud, en especial a las personas usuarias del SUS, que nos acompañaron con acciones y en la investigación. A Nathalia Rosa, que nos ayudó a traducir el neologismo "fazejamento" al español.

\section{REFERENCIAS BIBLIOGRÁFICAS}

1. Brasil. Emenda Constitucional No. 95. Diário Oficial da União [Internet]. 16 dez 2016 [citado 10 oct 2020]. Disponible en: https://tinyurl.com/ny43wdww.

2. Mariano CM. Emenda constitucional 95/2016 e o teto dos gastos públicos: Brasil de volta ao estado de exceção econômico e ao capitalismo do desastre. Revista de Investigações Constitucionais. 2017;4(1):259-281. doi: 10.5380/rinc.v4i1.50289.

3. CONASEMS. Brasil, aqui tem SUS [Internet]. 2020 [citado 28 nov 2020]. Disponible en: https://tinyurl.com/ cx5dmf37.

4. CONASEMS. Brasil, aqui tem SUS: Cooperação entre a prefeitura de Quissamã-RJ e a Universidade no enfrentamento da Covid-19 [Internet]. 2020 [citado 17 nov 2020]. Disponible en: https://tinyurl.com/zktwbhtt.

5. Garcia LP, Traebert J, Boing AC, Santos GFZ, Pedebôs LA, d'Orsi E, et al. O potencial de propagação da COVID-19 e a tomada de decisão governamental: uma análise retrospectiva em Florianópolis, Brasil. Revista Brasileira de Epidemiologia. 2020;23:e200091. doi: 10.1590/1980-549720200091.

6. Seixas TC, Merhy EE, Feuerwerker LCM, Espírito Santo TB, Slomp Junior H, Cruz KT. A crise como potência: os cuidados de proximidade e a epidemia pela Covid-19. Interface - Comunicação, Saúde, Educação. 2020;25(Supl.1):e200379. doi: 10.1590/interface.200379.

7. Caponi S. Covid-19 no Brasil: entre o negacionismo e a razão neoliberal. Estudos Avançados. 2020;34(99):209224. doi: 10.1590/s0103-4014.2020.3499.013.

8. Cruz KT, Coelho KSC, Vieira UP, Tavares BM, Slomp Junior H, Cinelli LP, et al. Relato de experiência: criação do grupo de trabalho multidisciplinar para o enfrentamento da COVID-19 na Universidade Federal do Rio de Janeiro - UFRJ campus Macaé. Boletim Ciência Macaé. 2020;1(1):80-90.

9. UFRJ-Macaé. GT Covid-19 UFRJ/Macaé [Internet]. 2020 [citado 14 nov 2020]. Disponible en: https:// gtcovid19.macae.ufrj.br.

10. Cruz KT, Coelho KSC. Relatório técnico do Grupo de Multidisciplinar de Enfrentamento da Covid-19 da UFRJ/ Macaé. 2020.

11. Sperandio N, Nascimento FTM, Monteiro LS, Coelho KSC, Guimarães ACC, Souza TO, et al. Interiorização da pandemia: panorama da COVID-19 no município de Macaé, Rio de Janeiro. Boletim Ciência Macaé. 2020;1(1):4-14.
12. Barros DM. Ações da gestão municipal para o enfrentamento da pandemia COVID 19 em Quissamã/ RJ [Internet]. Banco de Práticas e soluções em Saúde e Ambiente; 2020 [citado 28 nov 2020]. Disponible en: https://tinyurl.com/43cph2×3.

13. Barros DM, Gomes TCC, Merhy EE, Slomp Júnior H, Cruz KT, Coelho KSC. Relato da cooperação entre a Prefeitura de Quissamã e a Universidade Federal do Rio de Janeiro-campus Macaé para o enfrentamento da COVID-19 [Internet]. Banco de Práticas e soluções em Saúde e Ambiente; 2020 [citado 28 nov 2020]. Disponible en: https://tinyurl.com/43cph2x3.

14. Merhy EE, Cecílio LCO, Nogueira Filho RC. Por um modelo tecno-assistencial da política de saúde em defesa da vida: contribuiçäo para as conferências de saúde. Saúde em Debate. 1991;(23):83-89.

15. Franco TB, Bueno WS, Merhy EE. O acolhimento e os processos de trabalho em saúde: o caso de Betim, Minas Gerais, Brasil. Cadernos de Saúde Pública.1999;15(2):345-353. doi: 10.1590/S0102-311X1999 000200019 .

16. Feuerwerker LCM. Micropolítica y salud: producción del cuidado, gestión y formación. Porto Alegre: Editora Rede UNIDA, 2014.

17. Brossat A. Caixa de herramientas ou supermercado de ideias? Mnemosine. 2018;14(1):274-279.

18. Foucault M, Deleuze G. Los intelectuais y el poder. Microfísica del poder. Biblioteca de filosofia y história de las ciências. 4a ed. Rio de Janeiro: Edições Graal, 1984.

19. Merhy EE, Feuerwerker LCM. Novo olhar sobre as tecnologias de saúde: uma necessidade contemporânea. En: Merhy EE, Baduy RS, Seixas CT, Almeida DES, Slomp Junior $\mathrm{H}$, org. Avaliação compartilhada do cuidado em saúde: surpreendendo o instituído nas redes. Rio de Janeiro: Hexis; 2016. p. 59-72.

20. Instituto Brasileiro de Geografia e Estatística. Cidades e Estados: Quissamã [Internet]. 2020 [citado 17 nov 2020]. Disponible en: https://tinyurl.com/3h2ca4v3.

21. Silva AO. COVID-19: Um olhar diferenciado aos usuários com doenças crônicas não transmissíveis em tempos de pandemia no município de Quissamã/RJ. [Internet]. Banco de Práticas e soluções em Saúde e Ambiente; 2020 [citado 28 nov 2020]. Disponível em: https://tinyurl.com/43cph2×3.

22. Viana MPG. Estudo de soroprevalência de infecção por COVID-19 no município de Quissamã-RJ. [Internet]. Banco de Práticas e soluções em Saúde e Ambiente; 2020 [citado 28 nov 2020]. Disponible en: https:// tinyurl.com/43cph $2 \times 3$.

23. Brasil, Ministério da Saúde. Portaria GM no 414, de 18 de março de 2020. Diário Oficial da União [Internet]. 2020 [citado 20 oct 2020]. Disponible en: https://tinyurl. com/utshmj9x.

24. Brasil, Ministério da Saúde. Portaria SAS no 245, de 24 de março de 2020. Diário Oficial da União [Internet]. 
2020 [citado 10 oct 2020]. Disponible en: https://tinyurl. com/cvd7p6dd.

25. Brasil, Ministério da Saúde. Portaria GM no 1.269 de 18 de maio de 2020. Diário Oficial da União [Internet]. 2020 [citado 10 oct 2020]. Disponible en: https://tinyurl. com/333uvhkv.

26. Brasil, Ministério da Saúde. Portaria GM no 1.445, de 29 de maio de 2020. Diário Oficial da União [Internet]. 2020 [citado 10 oct 2020]. Disponible en: https://tinyurl. com/475p3jur.

27. Brasil, Ministério da Saúde. Portaria GM no 1.797, de 21 de julho de 2020. Diário Oficial da União [Internet]. 2020 [citado 10 oct 2020]. Disponible en: https://tinyurl. com/ynh4k852.

28. Rio de Janeiro, Secretaria de Estado da Saúde. Resolução SES n 2023, de 30 de março de 2020. Diário Oficial do Estado do Rio de Janeiro [Internet]. 2020 [citado 10 oct 2020]. Disponible en: https://tinyurl. com/4nfrxc9n.

29. Gomes DCM. Intervenções da Saúde Mental no município de Quissamã/RJ para o enfrentamento da pandemia COVID-19 [Internet]. Banco de Práticas e soluções em Saúde e Ambiente; 2020 [citado 28 nov 2020]. Disponible en: https://tinyurl.com/43cph2x3.

30. Guimarães ACC, Coelho KSC, Cruz KT, Souza BSO, Almeida JF, Coelho GF, et al. Comparative analysis of Covid-19 mortality in Brazil, Rio de Janeiro, Campos dos Goytacazes, Macaé, Cabo Frio and Rio das Ostras. medRxiv. 2020; 2020.09.17.20196444. doi: 10.1101/2020.09.17.20196444.

31. UFRJ/Macaé. Combate à COVID-19 na UFRJ-Macaé [Internet]. 2020 [citado 8 dic 2020]. Disponible en: https://tinyurl.com/k26dkfuz.

32. UFRJ/Macaé. Painel COVID-19 Norte Fluminense. [Internet]. 2020 [citado 8 dic 2020]. Disponible en: https://painelcovid19.macae.ufrj.br.

33. Chaves LA, Bossato HR, Amaral IBST, Cruz KT. Proposta de uma Central de Teleatendimento para pessoas em isolamento domiciliar durante a pandemia do coronavírus. Boletim Ciência Macaé. 2020;1(1):91-106.
34. Bondía JL. Notas sobre a experiência e o saber de experiência. Revista Brasileira de Educação. 2002;(19):20-28. doi: 10.1590/S1413-24782002000100003.

35. Lapoujade D. William James: a construção da experiência. São Paulo: N-1 Edicões; 2017.

36. Merhy EE. Planejamento como tecnologia de gestão: tendências e debates do planejamento em saúde no Brasil. En: Gallo E, Gonçalves RBM, Merhy EE, orgs. Razão e planejamento: reflexões sobre política, estratégia e liberdade. São Paulo: Hucitec, ABRASCO; 1995. p. 117-149.

37. Franco TB, Merhy EE. El reconocimiento de la producción subjetiva del cuidado. Salud Colectiva. 2011;7(1):9-20. doi: 10.18294/sc.2011.251.

38. Brasil, Conselho Nacional de Saúde. Recomendação no 22 de 09 de abril de 2020 [Internet]. 2020 [citado 10 oct 2020]. Disponible en: https://tinyurl.com/na44jmt3.

39. Barros DM, Batista SMS, Pereira SS, Nunes RM, Pessanha RBA, Ribeiro JCES, et al. Diálogos entre serviços: estratégias de integração e qualificação do cuidado em saúde - relato de experiência em Quissamã (RJ). En: Fernandes VR, Magalhães MG, D'Oliveira CBLC, Campos Filho E, Sergio JV, Silva JPV, et al., orgs. IdeiaSUS: saberes e práticas nos territórios do Sistema Único de Saúde. Rio de Janeiro: CEBES; 2020. p. 69-97.

40. Franco TB. O Cuidado à Saúde Mental em Tempos de Pandemia de Covid-19. Boletim da Sociedade de Reumatologia do Rio de Janeiro. 2020;XLVIII(176).

41. Slomp Junior $H$, Merhy EE, Seixas $C T$, Cruz KT, Bertussi DC, Baduy RS, et al. Mágica ou magia? Colegiados gestores no Sistema Único de Saúde e mudanças nos modos de cuidar. Interface - Comunicação, Saúde, Educação. 2019;23:e170395. doi: 10.1590/interface.170395.

42. Carvalho G. A saúde pública no Brasil. Estudos Avançados. 2013;27(78):7-26. doi: 10.1590/S0103-40142 013000200002.

43. Quissamã. Prefeitura Municipal de Quissamã [Internet]. 2020 [citado 19 nov 2020]. Disponible en: https://tinyurl.com/5y9cnh9w.

FORMA DE CITAR

Slomp Junior H, Coelho KSC, Barros DM, Franco TB, Cruz KT. Haciendo, planeando, "planhaciendo": una experiencia de pragmatismo municipal en la pandemia del Covid-19 en cooperación con la universidad. Salud Colectiva. 2021;17:e3341. doi: 10.18294/sc.2021.3341.

Recibido: 9 dic 2020 | Versión final: 8 feb 2021 | Aprobado: 10 feb 2021 | Publicado en línea: 28 may 2021

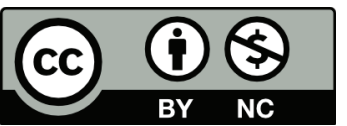

Esta obra está bajo una licencia de Creative Commons Reconocimiento-NoComercial 4.0 Internacional. Reconocimiento - Permite copiar, distribuir y comunicar públicamente la obra. A cambio, se debe reconocer y citar al autor original. No Comercial - Esta obra no puede ser utilizada con finalidades comerciales, a menos que se obtenga el permiso. 